\section{Herausforderungen für die Siedlungswasserwirtschaft}

Die Abwasserentsorgung steht aktuell vor einer Vielzahl von Herausforderungen, die sich gleichermaßen auf die Abwasserableitung und die Abwasserreinigung beziehen. Dabei geht es u. a. um die Anpassung der bestehenden Infrastruktur an neue Umweltbedingungen, um die Umsetzung gesetzlicher Vorgaben und um die Verbesserung bestehender Technologien zur Erfüllung des Gewässerund Ressourcenschutzes. Ein sehr umfangreicher Themenkatalog, der leicht einige Nummern der ÖWAW füllen könnte. Einigen Themen davon ist dieses Heft gewidmet.

Klimawandel und Global Warming sind Schlagworte, die heute in allen Lebensbereichen strapaziert werden. Wie sicher oder unsicher alle sich damit beschäftigenden Prognosen auch sein mögen, dieses Thema muss in der Planung der Infrastruktur zukünftig berücksichtigt werden. Die prognostizierte Zunahme an Starkregenereignissen kann dazu führen, dass in vielen Städten der Überflutungsschutz nur mehr unzureichend erfüllt werden kann. In diesem Zusammenhang haben Innsbrucker Wissenschaftler ein strategisches Planungstool entwickelt, mit dessen Hilfe neue Anpassungsstrategien und Technologien auf ihre Wirksamkeit getestet werden können. Konkret wird das Potenzial von Infiltrationsanlagen zur Kompensierung möglicher Effekte des Klimawandels untersucht.

Neben planerischen sind auch betriebliche Aufgaben der Siedlungsentwässerung von großem Interesse, wie z. B. die Kanalinspektion. Schon seit geraumer Zeit ist unbestritten, dass ihr in Hinblick auf den Betrieb (insbesondere für die Reinigung) eine wichtige Rolle zukommt. Zwei Aspekte werden diesbezüglich in diesem Heft von Wissenschaftlern der BOKU Wien behandelt.

Einerseits der finanzielle Aspekt, sind es doch die hohen dabei auflaufenden Kosten, die Kommunen veranlassen, die Reinigungsintervalle auszudehnen bzw. notwendige Reinigungsmaßnahmen aufzuschieben. Im ÖWAV-Regelblatt 22 wird zur Abhilfe eine vorausschauende Betriebsführung empfohlen, wofür eine einfache und schnelle Methode zur Erfassung des Verschmutzungsgrades des Kanals getestet worden ist - die Schacht-Zoom-Kamera, mithilfe derer erhebliche Einsparungen bei der Kanalreinigung erzielt werden können.

Andererseits müssen das Ergebnis einer Kanalinspektion und die damit verbundene Zustandserfassung - als wichtige Teile des integralen Kanalmanagements - zuverlässig und richtig sein. Über 50 \% falsche Erkennungsraten von Zuständen, wie sie in der Praxis vorkommen, dürfen nicht toleriert werden. In diesem Heft wird eine vereinfachte Methode der Zustandsklassifizierung ohne Berücksichtigung quantitativer Aspekte als Alternative zur aktuellen umfassenden Herangehensweise vorgestellt und anhand verfügbarer Inspektionsergebnisse praktisch eingesetzt.

Schließlich widmet sich ein Beitrag dieses Hefts einem qualitativen Abwasserthema, das heute zu Unrecht nicht allzu sehr im Fokus aktueller abwassertechnischer Aktivitäten steht, nämlich den Mineralölabscheidern. Die technische Gestaltung ihrer Leistung bzw. Wirksamkeit sowie ihre Überprüfung folgen bekannten Standards, deren Gültigkeit infrage gestellt werden muss, wenn heutzutage die Verwendung von biogenen Anteilen in Kraftstoffen gesetzlich vorgeschrieben wird. Mit praktischen Untersuchungen und Messergebnissen wird diese Feststellung untermauert.

Die Herausgeber sind überzeugt, dass die hier präsentierten Ergebnisse und Erfahrungen auf breites Interesse innerhalb der Fachwelt stoßen und für die zukünftigen abwassertechnischen Entwicklungen relevant sein werden.

\section{Priv.-Doz. DI Dr. Thomas Ertl}

Univ.-Prof. DI Dr. Raimund Haberl

Universität für Bodenkultur Wien

Department Wasser-Atmosphäre-Umwelt

Muthgasse 18

1190 Wien

thomas.ert|@boku.ac.at

raimund.haberl@boku.ac.at

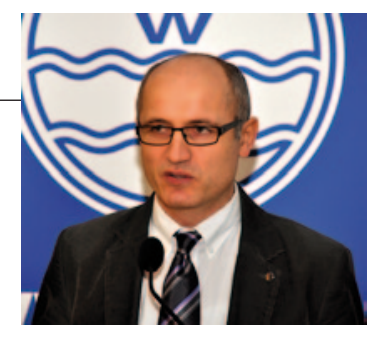

Priv.-Doz. DI Dr. Thomas Ertl

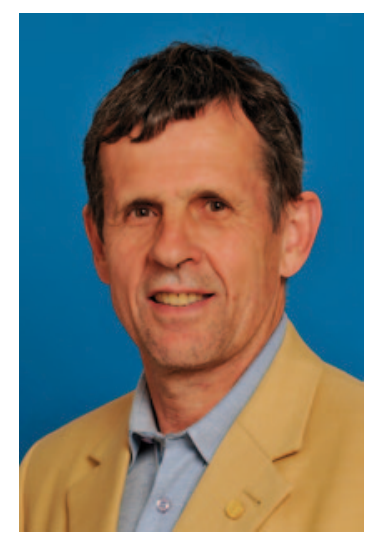

Univ.-Prof. DI Dr. Raimund Haberl 